http://jmscr.igmpublication.org/home/ ISSN (e)-2347-176x ISSN (p) 2455-0450 crossref DOI: https://dx.doi.org/10.18535/jmscr/v8i8.59

\title{
Post Burn Contracture Neck - Alternative Way of Airway Management
}

\author{
Authors \\ Dr Dinesh Sharma ${ }^{1}$, Dr Neeraj Sharma ${ }^{2}$ \\ ${ }^{1}$ Medical Officer (Anaesthesia), Civil Hospital Arki, Distt.- Solan, H.P. Pin- 173208, India \\ ${ }^{2}$ Senior Resident, Deptt. of Anaesthesia, YSPGMC Nahan, H.P. Pin 173001, India
}

\begin{abstract}
This case report encourages anaesthetists, to use alternative modes of securing endotracheal airway in patients of post burn contracture neck patients. Eighteen years old female patient of post burn contractures of neck was scheduled for contracture release and skin grafting. Airway assessment revealed severe restriction of mouth opening with limited restriction of neck movements.After preoxygenation, patient was induced with i/v propofol and oral intubation was attempted with intubating LMA. Successful ventilation achieved but endotracheal tube couldn't be inserted. Endotracheal intubation done with $7 \mathrm{~mm}$ CoETT with stylet using Video laryngoscope. Though fibreoptic bronchoscope guided intubation is gold standard and is the preferred technique and followed by most of anaesthetists, alternative methods of endotracheal intubation in case of difficult airways should also be considered helpful.
\end{abstract}

\section{Introduction}

Airway management during reconstructive surgery in Post Burn Contracture Neck patients is a great challenge for anaesthetist. Cervical motion may be limited in all directions and the sniffing position may be unobtainable due to severe mentosternal contracture. ${ }^{4}$ Along with restricted neck movements, patients may have limited mouth opening, distorted nasal anatomy, microstomia. Larynx and mandible may be distorted by underlying dense fibrous hypertrophic scar.

\section{Case Report}

A 18-year-old female patient weighing $40 \mathrm{~kg}$ was admitted with post burn contractures of neck and anterior chest wall, scheduled for contracture release and skin grafting. She had suffered burns 3 years earlier, while her clothes caught fire during cooking. She gave history of progressive thickening and scarring over burn area, after which she developed restricted neck movements up-down and sideways. She had SSG 3 times previously one year ago under sedation and LA.

\section{Airway Examination}

$>$ Mouth opening:- $2 \mathrm{FB} / 3 \mathrm{~cm}$

$>$ Protrusion of lower jaw:- can't be protruded

$>$ Facial deformity present - Rt. angle of mouth displaced downwards

$>$ Palate not arched

$>$ SMS Consistency - firm,

$>$ TMD - can't be assessed

$>$ Neck - short, contracture present on anterior aspect of neck 
Neck Movements - All restricted and Flexion at neck

Delilkan test - positive

Her vital signs were as follows:

Blood pressure 112/86 $\mathrm{mmHg}$ pulse rate $82 / \mathrm{min}$ respiratory rate $16 / \mathrm{min}$. Systemic examination was normal.

Hemoglobin was $10.9 \mathrm{~g} / \mathrm{dl}$

Difficult airway was predicted hence preoperative preparation with necessary masks, airways, endotracheal tubes, laryngeal mask airway (LMA's), stylets, bougie were kept ready. Airway plan was discussed thoroughly with plastic surgeon and ENT surgeon who were available for emergency tracheostomy, if necessary.Surgeons were told to be ready for release of contracture under ketamine and LA in case we could not secure airway

Patient was kept fasting for 8 hours. Pt. shifted to the OT at 9:30 am, identity was confirmed and monitors were attached for ECG, NIBP, Pulse oxymetry, temperature monitoring. Baseline vitals noted.IV Line secured with 18 gauge peripheral intravenous catheter in the left hand. In awake patient preoxygenation was successfully done ensuring a good face mask seal. In premedication: Inj. Fentanyl $50 \mathrm{mcg}$ I/V slow, Inj. Glycopyrrolate $0.2 \mathrm{mg} \mathrm{I} / \mathrm{V}$. , Inj. Ondansetron $4 \mathrm{mg} \mathrm{I} / \mathrm{V}, \mathrm{Inj}$. Ceftriaxone $1 \mathrm{gm} \mathrm{I} / \mathrm{V}$ given.

\section{Induction \& Intubation}

Surgeons were kept ready for release of neck contracture. Preoxygenation was done with $100 \%$ Oxygen for 3 minutes. Induction was done with Inj. Propofol 50mg I/V slow, then titrated till end point. Intubating LMA Size no. 4 was inserted and $\mathrm{B} / \mathrm{L}$ air entry confirmed.

After ensuring ventilation Inj. Atracurium 20mg $\mathrm{I} / \mathrm{V}$ given and ventilated for 3 minutes. Then tried to insert CoETT $7.0 \mathrm{~mm}$ ID through ILMA couldn't negotiate the tube. After proper suctioning Intubating LMA was taken out and Inj. Propofol $30 \mathrm{mg}$ i/v slow given. Endotracheal intubation done with $7 \mathrm{~mm}$ CoETT with stylet using Video laryngoscope. B/L air entry was checked and confirmed and ETT fixed at $21 \mathrm{~cm}$ mark. Inj. Dexamethasone $8 \mathrm{mg} \mathrm{I} / \mathrm{V}$ were given.

Maintenance of Anaesthesia was done with inhalational agents and intermittent atracurium, Injdiclofenac75mg, inj. Fentanyl 50mcg I/V, Inj PCM $1 \mathrm{gm}, \mathrm{I} / \mathrm{V}$ Fluids. Patient maintained $\mathrm{SpO}_{2}$ $98-100 \%$ and total length of surgery was $2 \mathrm{hrs} 30$ minutes. Vitals were stable throughout the surgery

\section{Extubation}

Muscle relaxation was reversed with neostigmine and glycopyrrolate. Awake extubation was done. Patient maintained $\mathrm{SpO}_{2}$ above $96 \%$ with oxygen with ventimask in propped up position. Kept patient in recovery for 30 minutes then shifted to ward.

\section{Post-op assessment}

Patient was fully conscious and had no complaint fresh complaints 2 hours after extubation.

\section{Discussion}

A pre-planned strategy should be there for intubation of the difficult airway. Such difficult airway preferably be secured while the patient is awake. FOB guided endotracheal intubation is Gold standard in PBC Neck patients ${ }^{[1]}$. Various case reports tell successful anaesthetic management of PBC neck in different way specially in airway management. The options advocated for airway management of such patients include:-

1) Awake fibreoptic - guided intubation. ${ }^{[1]}$

2) Video-laryngoscope guided intubation ${ }^{[2]}$

3) Use of LMA without intubation ${ }^{[3]}$

4) Use of intubating $\mathrm{LMA}^{[4]}$

5) Intubation without neuromuscular blocking agents.

6) Intubation with neuromuscular blocking agents after testing the ability to ventilate by mask/ supraglottic airway device, our preferred plan for this case.

7) Pre-induction neck scar release under local anesthesia and ketamine or sedation followed by direct laryngoscopy and intubation. ${ }^{[5]}$ 
8) Induction with ketamine and inhalational agents followed by DL examination - if unsuccessful give transverse incision over entire scar into skin and platysma to facilitate orotracheal intubation/LMA insertion, awake extubationat the end of surgery.

9) In nasal burns and microstomia, contracture of nose and oral cavity are to be released under nerve blocks first then assessing airway for method to be used for endotracheal intubation $^{[2]}$

10) Cervical (neurological damage) and lumbar (graft) epidural anaesthesia can be given, post-op analgesia can be maintained. ${ }^{[5,6]}$

We should inform the surgeons about the need of emergency scar release and/or emergency tracheostomy in case of CICO or failed intubation. We should have preformulated various intubation and extubation plans with adequate preparedness.

\section{Conclusion}

We should have a planned approach for securing airway. Plans A,B,C etc. should be clear to anaesthesia team members. Every plan must be discussed with surgeons and their role must be clear to them in maintaining airway if primary plans don't work.Rigid protocols should not be the best choice in view of variations in extent and severity of contractures as in our case.

\section{References}

1. Bokhari A, Benham SW, Popat MT. Management of unanticipated difficult intubation: A survey of current practice in the Oxford region. Eur J Anaesthesiol 2004;21:123-7.

2. Subramanyam: Anesthetic management of post-burn contracture chest with microstomia: Regional nerve blocks to aid in intubation Journal of Anaesthesiology Clinical Pharmacology | April-June 2015 | Vol 31 | Issue 2

3. Jasper, A. O. (2009). Anaesthetic management of post-burn contractures, a recurrent challenge from oil pipeline vandalization in Nigeria: a case report. Cases Journal, 2, 9141

4. Sarvesh, Shalini Chaudhary (2015) Difficult Airway Management In Patients With Severe Post Burn Contracture of Neck. Int J Anesth Res. 3(3), 93-95.

5. Gauri Arora. Anesthetic management of a case of multiple post-burn contractures/ International Journal of Biomedical Research 2016; 7(5): 303-305

6. BJA: British Journal of Anaesthesia, Volume 116, Issue 2, 1 February 2016, Pages 192-207. 